suspension, the measured cell voltage is different from that measured when they are both placed in the separate equilibrium solution (eqs). The SE is defined here as the sum of (1) the difference between the mixed potential of the indicator electrode (IE) in a suspension and the IE potential placed in the separated eqs; and (2) the anomalous liquid junction potential of the RE placed in the suspension. It is not the consequence of a boundary potential between the sediment and its eqs in the suspension potentiometric cells as is stated in the current definition of the SE.

Within Part A the measured cell potentials for suspension potentiometric cells are interpreted and explained in detail. With some former disagreements among investigations clarified, a new unambiguous operational definition of the SE is presented in Part $B$. It is defined as the difference in cell potential for two suspension potentiometric cells, one with both electrodes in the separated eqs and the other with both electrodes in the sediment or suspension. This potential difference is the sum of the change in the IE potential and the change in the liquid junction potential of the RE, when the electrodes are used for measurement, once in the sediment of the suspension and then in its eqs.

Tiil www.iupac.org/publications/pac/2007/7901/7901x0067.html

iin. www.iupac.org/publications/pac/2007/7901/7901x0081.html

\section{PAC is CrossRef Enabled}

CrossRef is a collaborative, cross-publisher referencelinking service that turns citations into hyperlinks, allowing researchers to navigate online literature at the article level. CrossRef is a wholly independent association of scholarly and professional publishers-large and small, commercial and nonprofit, traditional and nontraditional-that cooperate to provide reference links into and out of their electronic content. As such, CrossRef serves as the citation linking backbone for all scholarly literature online, facilitating and transforming the flow of information.

For its journal Pure and Applied Chemistry (PAC), IUPAC has been depositing DOIs (digital object identifiers) to CrossRef since January 2005. However, since January 2007, PAC has been participating in full by also offering outbound linking using the DOls received from CrossRef. These are links added to each reference published in each paper published in $P A C$. This way, papers that have cited a specific PAC paper can more easily link to that specific paper on the PAC website, but also papers published in PAC become more useful resources by offering links to cited references.

As a small publisher with only one journal, IUPAC made the decision to participate in CrossRef recognizing the benefit of increased accessibility and visibility. Given $P A C$ 's limited resources, the practical implementation took some time, but the engagement and will of Bohumir Valter was instrumental to the entire trans-

formation. His service to PAC, provided through Documents Data Services, supplements the existing work performed by the production editor Cheryl Wuzbacher and Cheryl Bush.

\section{More about CrossRef, see <www.crossref.org>}

\section{More about $P A C$, see <www.iupac.org/publications/pac>}
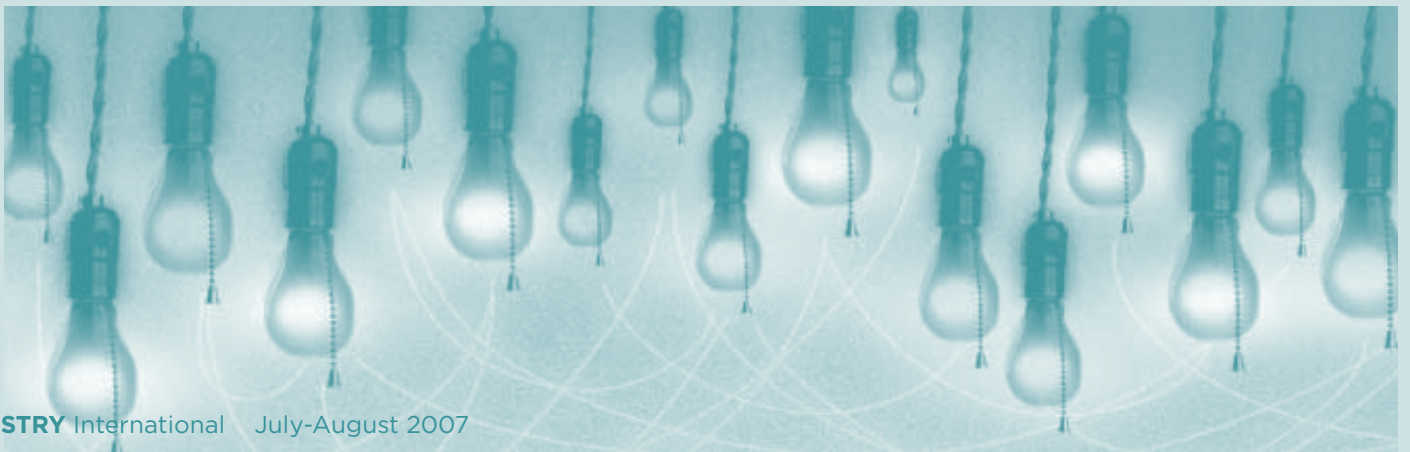